\title{
Multi-Resolution Geometric Fusion
}

\author{
Adrian Hilton and John Illingworth \\ Centre for Vision, Speech and Signal Processing \\ University of Surrey, Guildford GU25XH, UK \\ a.hilton@surrey.ac.uk \\ http://www.ee.surrey.ac.uk/Research/VSSP/3DVision
}

\begin{abstract}
Geometric fusion of multiple sets of overlapping surface measurements is an important problem for complete $3 D$ object or environment modelling. Fusion based on a discrete implicit surface representation enables fast reconstruction for complex object modelling. However, surfaces are represented at a single resolution resulting in impractical storage costs for accurate reconstruction of large objects.

This paper addresses accurate reconstruction of surface models independent of object size. An incremental algorithm is presented for implicit surface representation of an arbitrary triangulated mesh in a volumetric envelope around the surface. A hierarchical volumetric structure is introduced for efficient representation by local approximation of the surface within a fixed error bound using the maximum voxel size. Multi-resolution geometric fusion is achieved by incrementally constructing a hierarchical surface representation with bounded error. Results are presented for validation of the multi-resolution representation accuracy and reconstruction of real objects. Multi-resolution geometric fusion achieves a significant reduction in representation cost for the same level of geometric accuracy.
\end{abstract}

\section{Introduction}

Geometric fusion of multiple sets of overlapping surface measurements to form a complete 3D object or environment model has received considerable interest $[3,5,6,9,11,12$, $14,16,17]$. Reconstruction of 3D models of real objects is important for realistic computer generated imagery and accurate reverse engineering of CAD models. Two approaches have been proposed for fusion of multiple overlapping surface measurements into a single representation: mesh integration $[12,9,14,16]$ and volumetric fusion $[3,5,6,11,17]$. Mesh integration techniques enable fusion of multiple range images without loss of accuracy. In general mesh integration is sensitive to erroneous measurements which may cause catastrophic failure $[3,5]$. Recent research has addressed the sensitivity to erroneous measurements by incorporating visibility constraints[9]. Current mesh integration techniques are computationally expensive and do not allow reliable reconstruction from arbitrary surface measurements such as those obtained from a hand-held range sensor.

Volumetric fusion of surface measurements provides a general technique for model reconstruction from surface triangulations $[3,5,11]$. Measurements are combined into a single implicit surface representation which makes no assumptions about the geometry or topology allowing complex objects to be represented. This approach enables eliable reconstruction of complex object models without loss of accuracy [5]. Discrete implicit surface representation gives an order of magnitude reduction in computational cost [3]. Discrete representation causes a reduction in accuracy resulting in loss of surface detail and holes in the reconstructed model at crease edges or thin surface sections $[3,11]$. Previous discrete representations approximate the implicit surface at a single resolution for both complex geometric features and smooth regions. Representation costs are prohibitively expensive for accurate reconstruction of small surface features on large objects. This is a critical for reliable reconstruction of detailed environment models and accurate reverse engineering.

Multi-resolution geometric fusion addresses accurate reconstruction using a discrete implicit surface representation. The principal objective is accurate reconstruction independent of object size. A single resolution fusion algorithm is presented in section 2 for the construction of a discrete implicit representation from a set of arbitrary triangulated meshes. This approach enables fusion of surface measurements from both conventional range images and a hand-held range sensor. An efficient hierarchical structure for implicit surface approximation with bounded error is introduced in section 3. Unlike previous hierarchical field functions this representation provides efficient surface approximation based on local surface geometry for a volumetric envelope around the object surface. Multi-resolution fusion with bounded error is achieved by combining the hierarchical representation with the single resolution algorithm. Validation of the fusion algorithm and results for real objects are given in section 4 . 


\section{Single-Resolution Geometric Fusion}

This section presents an algorithm for fusion of an arbitrary set of triangulated meshes into a single resolution discrete implicit surface representation. Incremental triangleby-triangle transformation using the normal volume is used to construct a volumetric envelope around the surface. An efficient single resolution volumetric surface representation is obtained which is viewpoint independent unlike previous methods [3] is viewpoint independent. The incremental algorithm enables geometric fusion of surface measurements from both conventional range images or a hand-held range sensor.

\subsection{Volumetric Surface Representation}

An arbitrary topology closed manifold surface $S$ can be represented in implicit form as an iso-surface of a spatial field function, $f(\vec{x})$, where $\vec{x}=[x, y, z]$ is any point in Euclidean space, $R^{3}$. Thus we can represent a surface by defining the field function $f(\vec{x})$ as the signed distance from a point, $\vec{x}$, to the nearest point on the surface giving the iso-surface $f(\vec{x})=$ 0 for all points on $S$ and $f(\vec{x}) \neq 0$ elsewhere. A discrete volumetric representation can be implemented by uniform spatial subdivision into voxels cells and local planar approximation for voxels near the implicit surface, $f(\vec{x})=0$. Let us define a voxel grid based on a uniform spatial subdivision with voxel centres $\vec{c}_{\underline{\mathrm{i}}}=\left[x_{i}, y_{j}, z_{k}\right]$ given by:

$c_{\underline{\mathrm{i}}}=\left[\Delta x\left(i+\frac{1}{2}\right)+x_{m i n}, \Delta y\left(j+\frac{1}{2}\right)+y_{m i n}, \Delta z\left(k+\frac{1}{2}\right)+z_{m i n}\right]$

The discrete representation is defined in the range $\left[\vec{c}_{\text {min }}, \vec{c}_{\text {max }}\right]$ with uniform voxel resolution in each spatial dimension $\Delta c=\Delta x=\Delta y=\Delta z$. The number of voxel cells in each dimension is given by $\underline{N}_{V}=\left[\frac{\left(x_{\max }-x_{\min }\right)}{\Delta c}, \frac{\left(y_{\max }-y_{\min }\right)}{\Delta c}, \frac{\left(z_{\max }-z_{\min }\right)}{\Delta c}\right]$. The volume occupied by the voxel cell corresponding to centre $\vec{c}_{\underline{i}}$ denoted $V\left(\vec{c}_{\mathrm{i}}\right)=\left[x_{i} \pm \Delta c, y_{j} \pm \Delta c, z_{k} \pm \Delta c\right]$. For a given point $\vec{x}$ we can evaluate the corresponding voxel index $\underline{i}$ by:

$$
\underline{\mathrm{i}}=\left[\frac{\left(x-x_{\min }\right)}{\Delta c}, \frac{\left(y-y_{\min }\right)}{\Delta c}, \frac{\left(z-z_{\min }\right)}{\Delta c}\right]
$$

Volumetric surface representation is achieved by local planar approximation of the surface in the discrete voxel structure. Given an input triangulated mesh $M$ composed of a set ov vertices $P=\left[\vec{v}_{0}, \ldots, \vec{v}_{i}, \ldots, \vec{v}_{N_{P}-1}\right]$ and triangles $T=$ $\left[t_{0}, \ldots, t_{j}, \ldots, t_{N_{T}-1}\right]$ where $t_{j}=\left[\vec{v}_{r}, \vec{v}_{s}, \vec{v}_{t}\right.$. We assume that $M$ is a simply connected manifold triangulation with no selfintersections. Thus we can estimate the local surface normal for each triangle as $\vec{n}_{t_{j}}=\frac{\left(\vec{v}_{s}-\vec{v}_{r}\right) \times\left(\vec{v}_{t}-\vec{v}_{r}\right)}{\left\|\left(\vec{v}_{s}-\vec{v}_{r}\right) \times\left(\vec{v}_{t}-\vec{v}_{r}\right)\right\|}$. Vertex normals can be estimated by a weighted average of the adjacent triangle normals [15]:

$$
\vec{n}_{v_{r}}=\frac{\sum_{j \in r} w_{j} n_{t_{j}}}{\sum_{j \in r} w_{j}}
$$

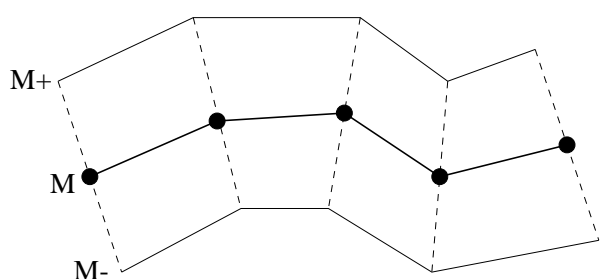

(a) Volumetric envelope

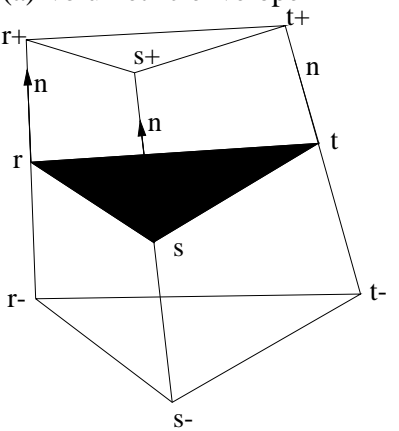

(b) Normal volume

Figure 1. Volumetric surface representation

A volumetric envelope is defined around the mesh $M$ which enables us to convert to an implicit volumetric representation. An offset surface $M^{\prime}$ for mesh $M$ is given by displacing each mesh vertex by a distance $d_{r}$ in the vertex normal direction such that $v_{r}^{\prime}=v_{r}+d_{r} \vec{n}_{r}$. If we let $d_{r}$ to be a constant offset distance $d_{\max }$ then the distance of all points on the offset surface $M^{\prime}$ is less than or equal to the offset distance $d_{\text {max }}$ from the original mesh $M$. The offset surface is a continuous mesh but may not be a simple manifold due to self-intersection. We can now define a volumetric envelope around $M$ by two offset meshes $M^{+}$and $M^{-}$such that each vertex is displaced by a distance $d_{\max }$ and $-d_{\max }$ in the normal direction respectively. The space enclosed by $M^{+}$and $M^{-}$is a closed volumetric envelope such that every point inside this region is less than $d_{\max }$ from the mesh $M$. This is illustrated for a cross section through a mesh in Figure 1(a).

For each triangle $t_{j}=\left[\vec{v}_{r}, \vec{v}_{s}, \vec{v}_{t}\right]$ in mesh $M$ the offset meshes $M^{+}$and $M^{-}$define a closed normal volume, $V_{N}\left(t_{r}\right)$, between $t_{j}^{+}=\left[\vec{v}_{r}^{+}, \vec{v}_{s}^{+}, \vec{v}_{t}^{+}\right]$and $t_{j}^{-}=\left[\vec{v}_{r}^{-}, \vec{v}_{s}, \vec{v}_{t}\right]$. The normal volume for a triangular element is illustrated in Figure 1(b). The concept of a volumetric envelope and normal volume or fundamental prism were recently used by Cohen et al.[2] to define an envelope for mesh simplification with bounded approximation error. Each side of the normal volume is a surface $S_{r s}$ constrained by three vectors the triangle edge $\vec{e}_{r s}=\vec{v}_{s}-\vec{v}_{r}$ and the corresponding vertex normals $\vec{n}_{r}$ and $\vec{n}_{s}$. If the vertex normals are equal $\vec{n}_{r}=\vec{n}_{s}$ then the surface $S_{r s}$ is a plane. In general if the normals are not equal we can satisfy the constraints by defining the surface $S_{r s}$ as a bilinear patch [2]. The volumetric envelope for mesh $M$ between offset meshes $M^{+}$and $M^{-}$is equivalent to the union of the normal volumes for all triangles 
$\bigcup_{r=0, N_{T}} V_{N}\left(t_{r}\right)$. Therefore, transforming the mesh $M$ to an approximate volumetric representation can be reduced to a incremental process of transforming the normal volume for each triangle, $t_{r}$ as follows:

1. Evaluate the normal volume $V_{N}\left(t_{r}\right)$.

2. Find the set of voxel centres inside the normal volume, $\left\{\ldots, \vec{c}_{\underline{i}}, \ldots\right\} \in V_{N}\left(t_{r}\right)$.

3. For all voxel centres inside the normal volume $\left\{\ldots, c_{i}, \ldots\right\}$ construct a local planar surface approximation from the nearest point on $t_{r}$ and the corresponding normal, $\left[\vec{p}_{\underline{\underline{1}}}, \vec{n}_{\underline{\mathbf{i}}}\right]$

To ensure that the normal volume, $V_{N} t_{j}$, encloses all points less than $d_{\max }$ from $t_{j}$ we can evaluate the vertex offset distance, $d_{r}$, as: $d_{r}=\frac{d_{m a x}}{\left(\vec{n}_{r} \cdot \vec{n}_{j}\right)}$. It is assumed that for any triangle in mesh $M$ the angle between a triangle and adjacent vertex normal is $\geq 90^{\circ}$ to avoid the degenerate case where the normal volume reduces to zero. To obtain a closed volumetric envelope for a voxel size $\Delta c$ it is necessary to set the offset distance $d_{\max } \geq \sqrt{3} \Delta c$. This gives a discrete field function $f(\vec{x})$ representation for all points with offset distance less than $\frac{\sqrt{3}}{2} \Delta c$ of the implicit surface $f(\vec{x})=0$ or mesh $M$.

\subsection{Single-Resolution Fusion Algorithm}

Fusion of multiple overlapping meshes, $M_{0}, \ldots, M_{N}$, can be achieved using the volumetric surface representation introduced in the previous section. For each mesh $M_{i}$ we can define a closed offset envelope between $M_{i}^{+}$and $M_{i}^{-}$with offset distance $d_{\max }$. Thus for each triangle we can define a $t_{r}$ a normal volume $V_{N}\left(t_{r}\right)$ and incrementally transform the mesh $M_{i}$ to a volumetric representation. In overlapping regions of surface measurements from different meshes the normal volumes will intersect provided the maximum measurement error $e_{\max } \leq d_{\max }$. If this condition is satisfied we can combine the field functions from different meshes to obtain a single volumetric representation. Fusion of multiple meshes requires an 'overlap' test to determine if surface measurements from different meshes in close spatial proximity correspond to the same or different regions of the measured object surface. Definition of a robust overlap test is critical for reliable surface reconstruction as discussed in previous work [5]. As in previous work geometric constraints are used to estimate if overlapping measurements correspond to the same surface region based on:

1. Spatial proximity: distance between overlapping measurements is less than the maximum distance $d_{\max }$.

2. Surface orientation: overlapping surface normals with the same orientation $n_{r} \cdot n_{t}>0$.

3. Measurement uncertainty: likelihood of measurement overlap based of estimates measurement uncertainty.

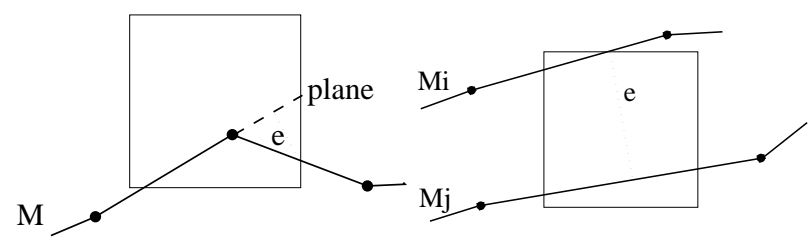

$\begin{array}{ll}\text { (a) Triangle boundary } & \text { (b) Multiple surfaces }\end{array}$

Figure 2. Volumetric representation error

Spatial proximity provides a coarse test of measurement overlap which has been used in previous work [3]. However, this test is unreliable for sharp edges and for surfaces in close proximity. Surface orientation enables reliable reconstruction of crease edges and thin surface sections for continuous implicit surface representation [5]. The use of measurement uncertainty depends on our ability to reliably estimate measurement uncertainty. For range images this can be estimated from the relative orientation of the surface normal and viewpoint. However, for a hand-held range sensor the viewpoint is continually changing and does not provide a reliable estimate of measurement uncertainty. If overlapping measurements are determined to correspond to the same surface region they may be combined according to a weighted average $[16,3,5]$ or maximum confidence [9].

\subsection{Performance of Single-Resolution}

Given a mesh $M$ we want to define bounds for the worst case approximation error of transforming $M$ into a discrete volumetric representation with voxel size $\Delta c$ according to the procedure introduced in 2.1. Two sources of discrete volumetric representation error occur. Firstly, voxel intersection with a triangle boundary in a non-planar region as illustrated in Figure 2(a). Secondly, voxel intersection with multiple mesh regions as illustrated in Figure 2(b). This may occur at crease edges and for thin surface regions. In planar mesh regions such as the interior of a triangle the discrete voxel representation is exact. The maximum approximation error, $e_{\max }$, occurs for multiple mesh intersections with a single voxel and is equal to the maximum voxel size, $e_{\max }=\sqrt{3} \Delta$. Reliable reconstruction of complex surfaces therefore requires a small voxel size.

The computation and memory cost of the volumetric representation depend on the number of voxels inside the offset envelope. For a uniform spatial subdivision of $N_{v}^{3}$ voxels the number of occupied voxels for a planar surface is $O\left(N_{v}\right)$, a spherical surface $O\left(N_{v}^{2}\right)$ and for a free-form surface may be even higher. Consequently, for a complex surface the computation and representation costs are proportional to the square (or higher power) of the voxel size. Conversely the representation accuracy is inversely proportional to the voxel size. Therefore, a uniform reduction in voxel size does not provide a satisfactory mechanism for efficient volumetric surface representation with bounded approximation error. 


\section{Multi-Resolution Geometric Fusion}

In this section we introduce a new multi-resolution discrete volumetric surface representation. The objectives of the multi-resolution approach are:

- Discrete implicit representation of a mesh $M$ with bounded approximation error $e_{\max } \leq \tau$ for a closed volumetric envelope $M \pm d_{\max }$.

- Accurate representation of surface features with a minimum voxel size $\Delta c \leq \frac{\tau}{\sqrt{3}}$.

- Efficient representation of smooth surface regions with voxels size $\geq \Delta c$.

- Fusion of multiple overlapping meshes, $\left\{M_{0}, \ldots, M_{N}\right\}$.

- Construction without building an intermediate single resolution representation at $\Delta c$.

- Representation cost independent of the size of the input triangulation.

The simplest algorithm for constructing the multiresolution representation with bounded approximation error is to first build a single uniform representation at the highest resolution $\Delta c \leq \frac{\tau}{\sqrt{3}}$. A multi-resolution representation with bounded error, $\tau$, can then be constructed by incrementally replacing the highest resolution voxels with corresponding lower resolution voxels while limiting the surface approximation error. However, single resolution representation is assumed to be prohibitively expensive in both computational and storage costs.

A multi-resolution representation which is independent of the size of the input triangulation is necessary to ensure that the cost does not continue to increase as new triangles that overlap surface regions already represented are added. If explicit references to the input triangles are stored in the volumetric representation then the representation cost is proportional to the number of input triangles. Cost proportional to the size of the input triangulation is prohibitively expensive for large sets of input triangulations with overlap.

In this section we introduce a procedure for sequential addition of new triangulated meshes into a hierarchical volumetric representation while maintaining an upper bound on the surface approximation error. This uses the normal volume approach introduced in section 2 to transform each triangle into a volumetric representation. For each voxel inside the normal volume at the highest resolution we determine the largest voxel size in the multi-resolution representation that gives a local surface approximation within the error bound, $\tau$.

\subsection{Multi-Resolution Volumetric Representation}

Octrees provide a hierarchical volumetric data structure for efficient representation of field functions for point clouds

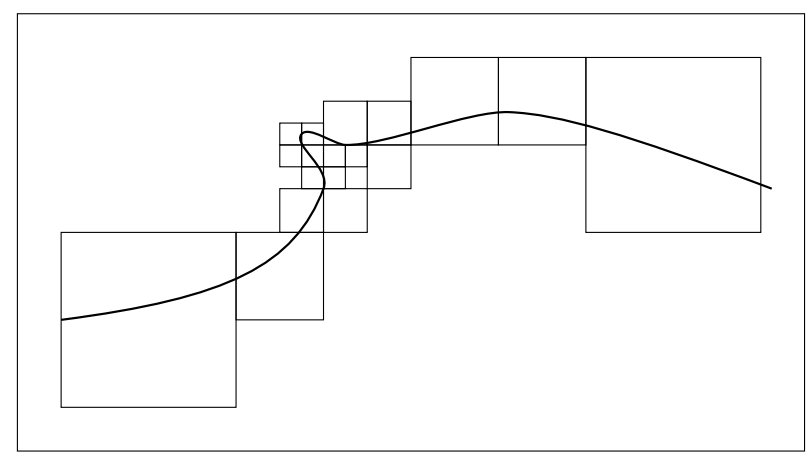

Figure 3. Multi-resolution representation

in $R^{3}$ [13]. In this section we extend the classical octree representation to enable efficient hierarchical surface representation. Octree splines [7] provide a technique for efficient implicit representation of an object surface, $S$, as a signed distance field function, $f(\vec{x})$. A discrete field function representation is stored for all points inside a bounding box around the surface, i.e. for the entire space occupied by the object. The field function is constructed from a set of points uniformly sampled on the surface, $S$. The hierarchical octree structure stores a discrete approximation of the field function with high spatial resolution near the surface and decreasing resolution with increasing distance from the surface. The cost of this representation with uniform re-sampling of the surface is therefore proportional to the surface area divided by the voxel resolution as discussed in section 2.3. This does not satisfy our objective of efficient multi-resolution volumetric implicit surface representation. The new multi-resolution volumetric surface representation presented in this section is based on an hierarchical spatial decomposition of a volumetric envelope around the surface. This is illustrated in Figure 3 which shows a cross section through the implicit surface, $f(\vec{x})=0$ with detailed features represented at high voxel resolution and smooth surface regions represented at low resolution. The surface approximation error is $\leq \tau$ for all points inside the volumetric envelope of size $d_{\max }$.

An octree volumetric representation can be defined with levels $l=0, \ldots, n_{l}$ such that the voxel size at level $l$ is given by $\Delta c^{l}=2^{l} \Delta c$ where $\Delta c$ is the voxel size at the highest resolution, $l=0$. For octree subdivision the voxel centres are given by:

$\vec{c}_{\underline{\mathrm{i}}}^{l}=\left[\Delta c^{l}\left(i+\frac{1}{2}\right)+x_{m i n}, \Delta c^{l}\left(j+\frac{1}{2}\right)+y_{m i n}, \Delta c^{l}\left(k+\frac{1}{2}\right)+z_{m i n}\right]$

The volume enclosed by each voxel is $V_{\underline{i}}^{l}=\vec{c}_{\underline{i}}^{l} \pm \Delta c^{l} / 2$. The octree subdivision gives a simple 8:1 correspondence between voxels at level $l$ and level $l+1$ for a volume $V_{\underline{i}}^{l+1}$ which is given by: 


$$
V_{\underline{\mathrm{i}}}^{l+1}=\bigcup_{j \in(2 \underline{\mathrm{i}}, 2 \underline{\mathrm{i}}+1)} V_{\mathrm{j}}^{l}
$$

As for the single-resolution volumetric surface representation we define a closed volumetric offset envelope for the mesh $M$. Let the offset distance be $d_{\max }^{0}=d_{\max }$ at level 0 . To ensure that at level $l$ the field function is defined for all points inside the offset envelope at level 0 we must set $d_{\text {max }}^{l} \geq \frac{\sqrt{3}}{2} \Delta c^{l}+d_{\text {max }}^{0}$. The normal volume $V_{N}^{l}\left(t_{j}\right)$ for a triangle $t_{j}$ at level $l$ is the volume between $t_{j}^{l+}$ and $t_{j}^{l-}$ where triangle vertex $\vec{v}_{r}^{l+}=\vec{v}_{r}+d_{\text {max }}^{l} \vec{n}_{r}$. Thus we can transform a mesh, $M$, to a single resolution volumetric representation at level $l$ by sequentially transforming each triangular element. The union of the normal volumes will form a closed volumetric envelope around the mesh $M$. Note the maximum surface approximation error for a single-resolution representation at level $l$ is given by $e_{\text {max }}^{l}=\sqrt{3} \Delta c^{l}$.

The problem now remains how to build an efficient multiresolution representation with bounded approximation error $\leq \tau$ for all points inside an offset distance $d_{\text {max }}$. We introduce a sequential procedure for adding each triangle $t_{j}$ in a mesh $M$ into the multi-resolution volumetric representation. Each voxel in the representation $\vec{c}_{\underline{1}}^{t}$ contains a local planar approximation, $\left[\vec{p}_{\underline{i}}^{l}, \vec{n}_{\dot{1}}^{l}\right]$, of the surface inside that voxel. For each triangle $t_{j}$ we first define the set of voxels at level 0 with centres inside the normal volume: $\left\{\ldots, \vec{c}_{\underline{\underline{i}}}^{0}, \ldots\right\} \in V_{N}^{0}\left(t_{j}\right)$. Next we determine the corresponding voxel for $c_{i}^{0}$ at level $l$ such that $\vec{c}_{\underline{i}}^{0} \in \vec{c}_{\underline{j}}^{t}$. The maximum approximation error $e^{l}$ for triangle $t_{j}$ at level $l$ is then computed. A simple function TEST can be defined to determine the maximum error, $e^{l}$ for approximation of triangle $t_{j}$ by the local planar surface approximation of voxel $c_{\underline{\mathrm{i}}}^{l}$.

1. Evaluate the nearest point on voxel plane, $\left[\vec{p}_{\underline{\mathbf{i}}}^{l}, \vec{n}_{\underline{\mathbf{1}}}^{l}\right]$ to each triangle vertex $\left(v_{r}, v_{s}, v_{t}\right)$ giving errors $\left(e_{r}^{l}, e_{s}^{l^{-}}, e_{t}^{l}\right)$.

2. Evaluate the maximum approximation error as: $e^{l}=$ $\max \left\{e_{r}^{l}, e_{s}^{l}, e_{t}^{l}\right\}$

If this procedure is repeated recursively for corresponding voxels at levels $l=1, \ldots, N_{l}-1$ we can determine the maximum level in the existing representation for which $e^{l}<\tau$. This provides a mechanism for transforming a triangle $t^{j}$ into a multi-resolution discrete volumetric approximation with bounded error. Repeating this process sequentially for each triangle in mesh $M$ will result in a discrete multiresolution volumetric representation. The volumetric representation is defined for an offset envelope, $d_{\max }$, around the mesh $M$ with bounded approximation error $\tau$.

\subsection{Multi-Resolution Fusion Algorithm}

In this section we introduce a procedure for fusion of multiple overlapping triangulated meshes into a single multi- resolution representation. Fusion of multiple overlapping meshes combines the single-resolution fusion algorithm introduced in section 2.2 with the multi-resolution representation presented in the previous section. We apply a bottomup procedure for adding new voxels into a hierachical representation which ensures that the maximum error remains bounded $\leq \tau$. The bottom-up procedure starts at the lowest level in the voxel hierarchy $l=0$ and tests the approximation error for $l=0 \ldots, n_{l}-1$. This bottom-up procedure is cautious as the first priority is ensure that the maximum approximation error remains bounded.

An alternative is to use a greedy top-down algorithm to approximate new triangles at the highest possible level in the multi-resolution representation and to refine the representation as new triangles are introduced. A top-down approach is desirable as it gives a considerable reduction in the computational cost of building the multi-resolution representation. However, it has been found that the greedy top-down approach does not allow bounded approximation error. Therefore, a more expensive bottom-up algorithm has been developed.

For a set of meshes, $\left\{M_{i}\right\} i=0, \ldots, N_{M}-1$, we transform each triangle $t_{j}$ into a discrete volumetric approximation using the normal volume approach as follows:

1. Evalutate the normal volume at level zero: $V_{N}^{0}\left(t_{j}\right)$.

2. Find the set of voxel centers inside the normal volume: $\left\{\ldots, \vec{c}_{\underline{\underline{i}}}^{0}, \ldots\right\} \in V_{N}^{0}\left(t_{j}\right)$.

3. For each voxel inside $\vec{c}_{\underline{i}}^{0}$ introduce a local planar approximation of triangle $t_{j}$ at the highest level, $l$, for which $e^{l}<\tau$ and $\vec{c}_{\underline{\mathrm{i}}}^{0} \in \vec{c}_{\mathrm{k}}^{l}$ using function ADD.

Steps 1 and 2 above are the same as for constructing a single resolution representation. A recursive insertion procedure, ADD, is introduced at step 3 to approximate $t_{j}$ at the highest level in the octree which gives a representation with bounded error. The following procedure is used to test the addition of new voxels $\vec{c}_{\dot{i}}^{l}$ into the representation at level $l$ to approximate $t_{j}$ or recursively to test the addition at the next level, $l+1$. Note the recursive procedure is a bottom-up search to find the highest level in the existing representation that gives bounded local approximation error. However, the addition of new voxels is cautious. If all sub-voxels at level $l$ in the existing representation are empty for a corresponding voxel at level $l+1$ then the new triangle is represented at level $l$. Function ADD for recursive multi-resolution triangle approximation is implemented as follows:

1. Find the correpsonding voxel at level $l+1$ where $\vec{c}_{\underline{\mathrm{i}}}^{l} \in V_{\underline{\mathrm{j}}}^{l+1}$.

2. If $\vec{c}_{\underline{j}}^{l+1}$ is occupied by a planar approximation $\left[\vec{p}_{\underline{\mathrm{j}}}^{l+1}, \vec{n}_{\underline{\mathrm{j}}}^{l+1}\right]$, TEST the maximum approximation error, $e^{l+1}$ for $\vec{c}_{\dot{1}}^{l}$ : 
(a) If $e^{l+1}<\tau$ then FUSE $\vec{c}_{\underline{\underline{i}}}^{l}$ with $\vec{c}_{\underline{j}}^{l+1}$

(b) Else If $e^{l+1}>\tau$ then INSERT $\bar{c}_{\underline{\underline{i}}}^{l}$ at level $l$.

3. Else If $\vec{c}_{\underline{j}}^{+1}$ is empty:

(a) Find the set of occupied sub-voxels at level $l$ inside $\vec{c}_{\underline{j}}^{l+1}$ : $\left\{\ldots, \vec{c}_{\underline{r}}^{l}, \ldots\right\} \in V_{\underline{j}}^{l+1}$.

(b) If there are no occupied sub-voxels INSERT $\vec{c}_{\underline{1}}^{l}$ at level $l$.

(c) Else If the number of occupied sub-voxels $\geq 0$ :

i. FUSE the sub-voxels $\left\{\ldots, \vec{c}_{\underline{r}}^{t}, \ldots\right\}$ to form a new candidate voxel: $\vec{c}_{\mathrm{j}}^{l+1}$.

ii. TEST approximation error of $\vec{c}_{\underline{j}}^{l+1}$ :
A. If $e^{l+1}<\tau$ ADD $\vec{c}_{\underline{j}}^{l+1}$ and Remove sub- voxels.
B. Else if $e^{l+1}>\tau$ INSERT $\vec{c}_{\underline{\mathrm{i}}}^{l}$ at level $l$.

The above algorithm uses the following sub-procedures Insert, Remove, Fuse, Test and AdD. Insert and ReMOVE modify the current representation by inserting or deleting voxels from the structure repspectively. FUSE uses the fusion algorithm defined for single-resolution volumetric data to combine data for overlapping surface measurements. TEST evaluates the maximum approximation error for triangle $t_{j}$ as defined in the previous section. ADD is a recursive call to the same procecedure for a voxel at the next highest level $l+1$. This enables a corresponding voxel at level $l+1$ to approximate multiple sub-voxels at level $l$ provided the bound on the approximation error is satisfied. Approximation of multiple sub-voxels at a higher level gives a reduction in representation cost. Note the bottom-up hierarchical approximation allows a new voxel at level 0 which will always give a surface approximation error less than $\tau$. Lower level voxels are only replaced at a higher level if the approximation error is bounded.

The procedure introduced above enables fusion of multiple meshes into an efficient multi-resolution volumetric representation. For multiple overlapping meshes to be integrated correctly the maximum measurement error in the input mesh must be less than the offset distance, $d_{\text {max }}$, at level $l=0$. This condition ensures that the normal volumes for overlapping surface measurements intersect and can be fused into a single representation. An equivalent assumption is made for the single-resolution volumetric fusion.

Single resolution implicit surface polygonisation $[8,4]$ has been used throughout this work to reconstruct an explicit triangulated model for visualisation. Future work will address multi-resolution adaptive polygonisation [1] to obtain an efficient triangulation.

\section{Results}

In this section we first verify the validity of the multiresolution representation and fusion algorithm introduced in section 3. Results are then presented for the use of multiresolution geometric fusion for reconstruction of 3D surface models of real objects. Surface measuremetns are obtained using both a conventional range image sensor and a hand-held range sensor. A comparison is given of the representation cost for single and multi resolution approaches.

\subsection{Validation of Multi-Resolution Representation}

To verify the accuracy of the multi-resolution representation we can construct a volumetric representation for a single mesh $M$. The distance between the original mesh and the implicit surface, $f(\vec{x})=0$, gives a measure of the representation error. The error at every vertex in the original mesh is evaluated and used to compute the root mean square (rms) and maximum representation error. Note the representation accuracy is expected to be lowest at the mesh verticies. Volumetric representation errors for a single input mesh are given in Table 1 for single-resolution and Table 2 for multiresolution resolution. Throughout this paper the error tolerance for multi-resolution representation is equal to the maximum error at the lowest resolution $\tau=\sqrt{3} \Delta c$. The following observation can be made from these results. The representaion error for multi-resolution representation is bounded $\leq \tau$. There is an order of magnitude reduction in representation cost between single and multi resolution for the same maximum error. Although there is a corresponding increase in the rms error for multi-resolution. For single-resolution the representation cost is approximately proportional to the square of the resolution. For multi-resolution representation cost reduction for different numbers of levels depends on the level of surface detail, i.e. for accurate representation of the edge voxels at the highest resolution are required. Figure 4 shows the reconstructed model of the edge and sphere for single and multi resolution sphere. The edge approximation accuracy is approximately equal for single and multi resolution. However, the surface is smoother for single resolution as expected from the lower rms error.

\subsection{Validation of Multi-Resolution Fusion}

The representation accuracy for fusion of multiple overlapping meshes is verified by comparing the error for fusion at single and multi resolution. Results are presented in Table 3 for two simple objects captured using a hand-held range sensor. A factor 3-5 reduction in representation cost is achieved for multi-resolution fusion with three or five levels. The maximum representation error remains bounded $\leq \tau$. The rms representation error is approximately double for five levels compared to three levels. 


\begin{tabular}{|c||c|c|c|c|c|c|}
\hline & input & size & res. & mem. & rms & max. \\
& $N_{T}$ & $N_{V}$ & $\Delta c$ & $(\mathrm{Mb})$ & $\% \Delta c$ & $\% \Delta c$ \\
\hline \hline Edge & 9660 & $200^{3}$ & 0.33 & 2.1 & 1 & 30 \\
& & $100^{3}$ & 0.67 & 0.5 & 1 & 16 \\
& & $50^{3}$ & 1.3 & 0.1 & 13 & 46 \\
\hline Sphere & 1490 & $200^{3}$ & 0.01 & 6.8 & 0.2 & 0.7 \\
& & $100^{3}$ & 0.02 & 1.7 & 0.2 & 8 \\
& & $50^{3}$ & 0.04 & 0.5 & 0.3 & 4 \\
\hline
\end{tabular}

Table 1. Single-resolution error

\begin{tabular}{|c||c|c|c|c|c|c|}
\hline & input & lev. & & mem. & rms & max. \\
& $N_{T}$ & $n_{l}$ & $\Delta c^{0}$ & $(\mathrm{Mb})$ & $\% \Delta c$ & $\% \Delta c$ \\
\hline \hline Edge & 9660 & 3 & 0.33 & 0.6 & 12 & 70 \\
& & 5 & & 0.4 & 12 & 90 \\
& & 8 & & 0.3 & 37 & 136 \\
\hline Sphere & 1490 & 3 & 0.01 & 0.5 & 1 & 3 \\
& & 5 & & 0.05 & 3 & 18 \\
& & 8 & & 0.04 & 40 & 63 \\
\hline
\end{tabular}

Table 2. Multi-resolution error $N_{V}=200^{3}$
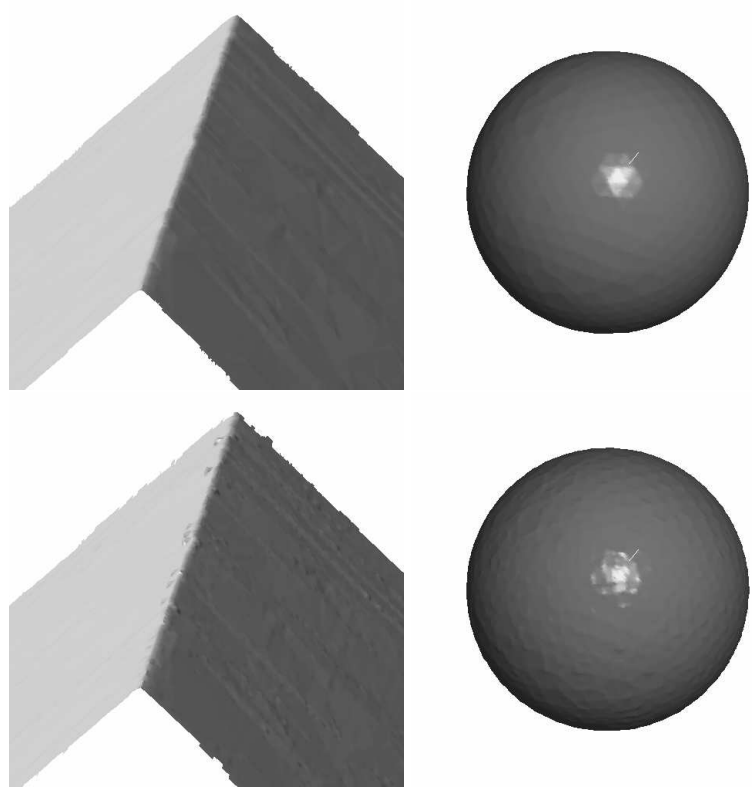

Figure 4. Reconstruction for single(top) \& multi(bottom $n_{l}=5$ ) representation

\begin{tabular}{|l||c|c|c|c|c|c|}
\hline & input & lev. & res. & mem. & rms & max. \\
$N_{T}$ & $n_{l}$ & $\Delta c$ & $(\mathrm{Mb})$ & $\% \Delta c$ & $\% \Delta c$ \\
\hline \hline Cube & $14 \mathrm{~K}$ & 1 & 0.32 & 22 & - & - \\
& & 3 & & 7 & 7 & 93 \\
& & 5 & & 4 & 13 & 115 \\
\hline Sphere & $32 \mathrm{~K}$ & 1 & 0.2 & 48 & - & - \\
& & 3 & & 10 & 25 & 87 \\
& & 5 & & 9 & 56 & 137 \\
\hline
\end{tabular}

Table 3. Multi-resolution fusion $N_{V}=400^{3}$

\begin{tabular}{|l||c|c|c|c|c|c|}
\hline & input & Size & res. & \multicolumn{3}{|c|}{ Memory Usage(Mb) } \\
& $N_{T}$ & $N_{V}$ & $\Delta c$ & $n_{l}=1$ & $n_{l}=3$ & $n_{l}=5$ \\
\hline \hline bunny & $40 \mathrm{~K}$ & $400^{3}$ & 0.4 & 21 & 7 & 6 \\
\hline soldier & $175 \mathrm{~K}$ & $400^{3}$ & 0.4 & 36 & 13 & 12 \\
\hline fan & $50 \mathrm{~K}$ & $400^{3}$ & 0.84 & 41 & 11 & 9 \\
\hline dwarf & $420 \mathrm{~K}$ & $400^{3}$ & 1.2 & 83 & 17 & 8 \\
\hline
\end{tabular}

Table 4. Fusion for complex objects
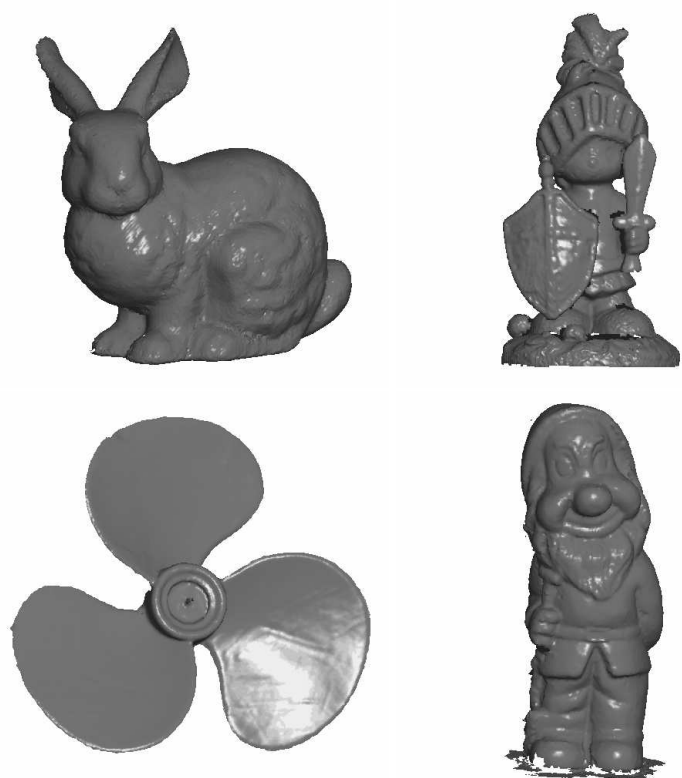

Figure 5. 3D models for complex objects

\subsection{D Model Reconstruction}

This section presents results for the multi-resolution fusion of surface measurements for complex real objects. Table 4 gives the representation cost for four objects for fusion with different numbers of levels. A factor three reduction in representation cost is obtained for multi-resolution fusion. Figure 5 shows the reconstructed 3D models for multi-resolution fusion with $n_{l}=3$. There is no visible reduction in the quality of the reconstructed model with the multi-resolution fusion approach. The bunny and soldier models were constructed from multiple range images from Cyberware[16] and NRC[10] scanners. The fan and dwarf models were constructed from hand-held range sensor data collected using the 3D Scanners, ModelMaker system. 


\section{Conclusions}

A single-resolution geometric fusion algorithm has been presented which allows volumetric implicit surface representation of a set of triangulated meshes. An incremental triangle-by-triangle procedure is introduced for converting an arbitrary mesh to a volumetric representation. The normal volume is used for efficient transformation of each triangle to a local volumetric representation. The union of the normal volumes form a volumetric offset envelope around the surface. This approach has the advantage over previous viewpoint based techniques that the volume is independent of the surface orientation allowing efficient representation. This algorithm enables fusion of surface measurements from both conventional range images and a hand-held range sensor. Measurements from a hand-held range sensor are a series of unstructured stripes with overfolding.

A hierarchical octree structure has been developed for efficient volumetric surface representation with bounded approximation error. The surface is locally represented by the largest voxel size with a planar patch that fits the surface measurements within an error tolerance. This enables efficient approximation for a volumetric envelope around the surface. Previous hierachical implicit surface representations [1,7] approximated the field function throughout the entire region of space occupied by the object. Results demonstrate that the hierarchical approximation achieves an order of magnitude reduction in representation cost with accurate represention of local surface geomtry for complex features.

Multi-resolution geometric fusion is achived by combining the hierachical volumetric surface representation with the incremental surface to volume transformation. A cautions bottom-up algorithm is introduced to obtain efficient surface representation whilst maintaining a tolerance on the surface approximation error. This algorithm gives computational cost approximately equal to the single resolution reconstruction at the highest resolution. Results demonstrate that this approach achieves a significant reduction in representation cost without a significant reduction in accuracy. Multi-resolution reconstruction of real object models using surface measurements from both range images and a hand-held range sensor gives a factor three reduction in representation cost. Further research is required to identify more efficient algorithms for constructing the multi-resolution representation and achieving further reductions in representation costs.

\section{Acknowledgements}

This reasearch was supported by EPSRC Grant GR/K04569 'Finite Element Snakes for Depth Data Fusion'. The authors wish to thank 3D Scanners Ltd,UK for testing and feedback of fusion algorithms. In particular Peter Champ who identified limitations of single resolution fusion.

\section{References}

[1] J. Bloomenthal. Polygonization of implicit surfaces. Computer Aided Geometric Design, 5:341 -355, 1988.

[2] J. Cohen, A. Varshney, D. Manocha, G. Turk, H. Weber, P. Agarwal, F. Brooks, and W. Wright. Simplifi cation envelopes. In SIGGRAPH, pages 119-128, 1996.

[3] B. Curless and M. Levoy. A volumetric method for building complex models from range images. In Computer Graphics Proceedings, SIGGRAPH, 1996.

[4] A. Hilton, A. Stoddart, J. Illingworth, and T. Windeatt. Marching triangles: Range image fusion for complex object modelling. In International Conf. on Image Processing, pages 381-384. Lusanne, 1996.

[5] A. Hilton, A. Stoddart, J. Illingworth, and T. Windeatt. Reliable surface reconstruction from multiple range images. In 4 th European Conference on Computer Vision, pages 117-126. Springer, 1996.

[6] H. Hoppe, T. DeRose, T. Duchamp, J. McDonald, and W. Stuetzle. Surface reconstruction from unorganised points. Computer Graphics, 26(2):71-77, 1992.

[7] S. Levallee and R. Szeliski. Recovering the position and orientation of free-form objects from image contours using 3d distance maps. IEEE Trans. Pattern Analysis and Machine Intelligence, 17(4):378-390, 1995.

[8] W. Lorensen and H. Cline. Marching cubes: A high resolution 3d surface construction algorithm. Computer Graphics, 21(4):163-169, 1987.

[9] R. Pito. Mesh integration based on co-measurements. In International Conf. on Image Processing, pages 397-400, 1996.

[10] M. Rioux. Laser range fi nder based on synchronized scanners. Applied Optics, 23(21):3837-3844, 1984.

[11] G. Roth and E. Wibowo. A fast algorithm for making meshmodels from multiple-view range data. In Proceedings of the 1995 Robotics and Knowledge Based Systems Workshop, pages 349-355, 1995.

[12] M. Rutishauser, M. Stricker, and M. Trobina. Merging range images of arbitrarily shaped objects. In Proceedings of IEEE Conference on Computer Vision and Pattern Recognition, pages 573-580, 1994.

[13] H. Samet. The design and analysis of spatial data structures. Addison-Wesley, 1990.

[14] M. Soucy and D. Laurendeau. A general surface approach to the integration of a set of range views. IEEE Trans. Pattern Analysis and Machine Intelligence, 14(4):344-358, 1995.

[15] G. Taubin. Estimating the tensor of curvature of a surface from a polyhedral approximation. In Proccedings IEEE International Conference on Computer Vision, pages 902-907, 1995.

[16] G. Turk and M. Levoy. Zippered polygon meshes from range images. In Computer Graphics Proceedings, SIGGRAPH, 1994.

[17] M. Wheeler. Automatic modeling and localization for object recognition. In Ph.D. Thesis, Carnegie Mellon University, CMU-CS-96-188, 1996. 